\title{
Transient twinkle perception is induced by sequential presentation of stimuli that flicker at frequencies above the critical fusion frequency
}

\author{
Yutaka Nakajima $^{1} \cdot$ Yutaka Sakaguchi $^{1}$
}

Published online: 22 July 2015

(C) The Psychonomic Society, Inc. 2015

\begin{abstract}
The critical fusion frequency (CFF) is a threshold that represents the temporal limits of the human visual system. If two flickering stimuli with equal subjective luminances are presented simultaneously at different locations, the CFF is the temporal frequency above which they cannot be distinguished. However, when the stimuli are presented sequentially at the same position, a transient twinkle can be perceived around the moment of the changeover. To investigate the mechanism underlying this transient twinkle perception (TTP), we independently manipulated the luminance contrast and temporal frequency of the flicker, as well as the interstimulus interval (ISI). We found that TTP was greater as the luminance step was larger, was stably perceived for flicker frequencies up to $200 \mathrm{~Hz}$, and was robust for all ISIs if flicker frequencies were below $250 \mathrm{~Hz}$. For 250 - and $300-\mathrm{Hz}$ flickers, TTP was attenuated in conditions in which one-frame and two-frame ISIs were inserted. These results can be explained by a simple filtering model: TTP occurs if the temporal change in the weighted moving average of stimulus luminance exceeds a certain threshold. TTP gives additional evidence that the human visual system can detect the transient change of flicker stimuli at much higher temporal frequencies than the $\mathrm{CFF}$, by an averaging mechanism of luminance.
\end{abstract}

Keywords Critical fusion frequency $\cdot$ Moving averaging · Temporal processing $\cdot$ Transient twinkle perception

Yutaka Nakajima

ytk.nakajima@gmail.com

1 Laboratory of Human Informatics, Graduate school of Information Systems, University of Electro-Communications, 1-5-1, Chofugaoka, Chofu-shi, Tokyo 182-8585, Japan
The critical fusion frequency (CFF) represents a temporal resolution limit of our visual processing. When we look at a stimulus flickering at a frequency higher than the CFF (above-CFF flicker), we only perceive a stationary field. Though the sensitivity to flicker varies depending on retinal illuminance (Kelly, 1961), eccentricity (Rovamo \& Raninen, 1984), stimulus size, and the duty ratio of the flicker (Emoto \& Sugawara, 2012), the CFF is generally around $60 \mathrm{~Hz}$ (explaining why the refresh rate of consumer displays is set at $60 \mathrm{~Hz}$ or higher). On the other hand, some studies have reported neural entrainment to above-CFF stimuli. For example, steady-state visually evoked potentials have been observed for above-CFF stimuli (Herrmann, 2001; Lyskov, Ponomarev, Sandstrom, Mild, \& Medvedev, 1998; RamosJúnior, Celino, Rodor, Ribeiro, \& Muller, 2011). In addition, a single flash of a half sinusoid can be detected at up to $600 \mathrm{~Hz}$ (Levinson, 1968).

Recently, an interesting phenomenon relevant to aboveCFF stimuli has been reported: When two different aboveCFF stimuli are sequentially presented, a transient flash is perceived (Cheadle, Parton, Muller, \& Usher, 2011; van Diepen, Born, Souto, Gauch, \& Kerzel, 2010). In these studies, two stimuli were presented sequentially at the same position; one was the "flicker stimulus," in which two differentluminance frames were alternatively presented, and the other was the "continuous stimulus," in which all frames had identical luminances. These stimuli were presented on a CRT monitor at above-CFF refresh rates. ${ }^{1}$ As a result, the flickering frequencies were below (e.g., $50 \mathrm{~Hz}$ ) and above (e.g., $100 \mathrm{~Hz}$ )

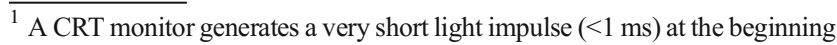
of every frame. Thus, for most of the frame, no light is physically emitted, even when the bright frames are presented synchronously with the v-sync of a CRT. This means that the "continuous stimuli" are technically not presented continuously, although for convenience we call this type of stimulus "continuous" to differentiate them from the "flicker" stimuli.
} 
the CFF for the flicker and continuous stimuli, respectively. The subjective luminances of these stimuli were matched so that observers could not differentiate them when they were presented at the separate locations. When these stimuli were presented at the same location (Fig. 1a), however, a transient "twinkle" was occasionally perceived (Cheadle et al., 2011; van Diepen et al., 2010). We call this phenomenon "transient twinkle perception" (TTP, Fig. 1b). Similar phenomena had been mentioned in classic studies with a point light (Bird \& Mowbray, 1969; Forsyth \& Brown, 1961; Mowbray \& Bird, 1969; Sen, 1964).

Reports have shown that the perceived luminance of the above-CFF flicker was identical to that of a non-flickering stimulus whose luminance was matched to the averaged luminance of the above-CFF flicker (Cheadle et al., 2011; van

(a)
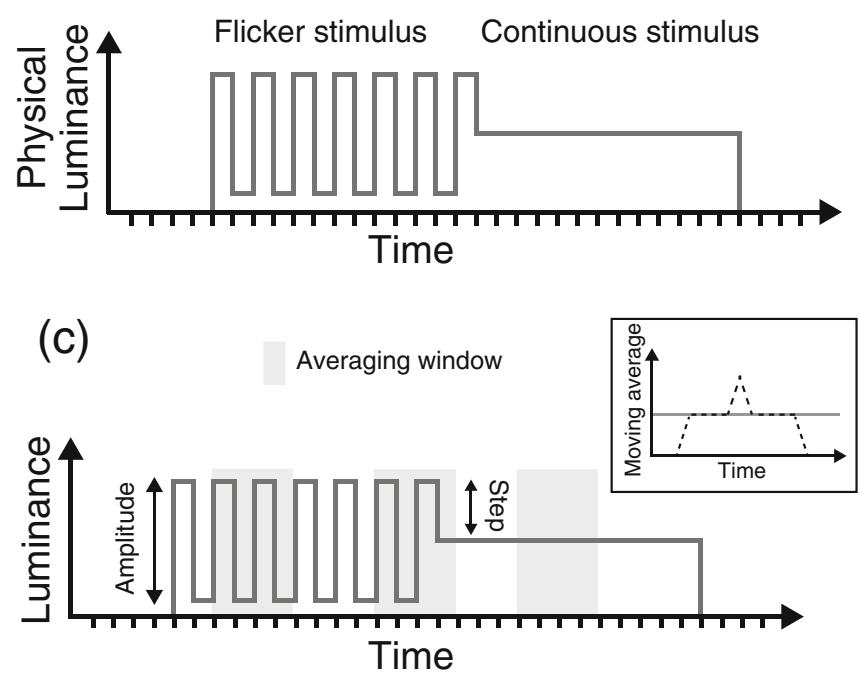

(e)

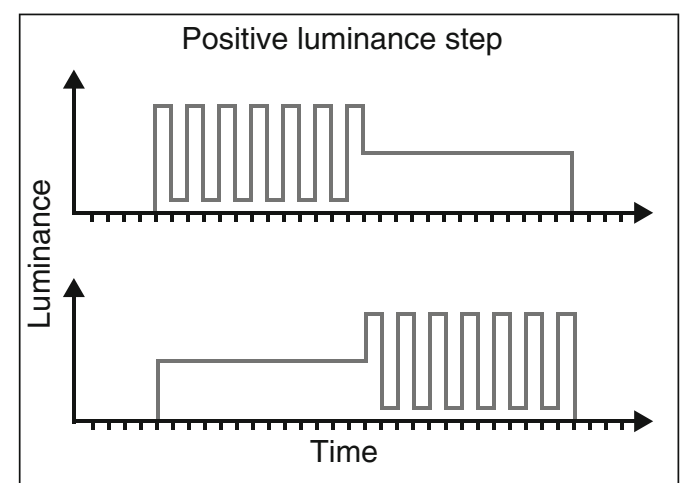

Fig. 1 Transient twinkle perceived when continuous and flicker stimuli are presented sequentially. (a) A flicker stimulus and a continuous stimulus are presented sequentially without an interstimulus interval. (b) Even if these stimuli have the same overall perceptual luminance, a brief luminance change (i.e., a transient twinkle) can be perceived just around the time of transition. (c) When the moving average is calculated for the stimulus in panel a with an averaging window, the average at the
Diepen et al., 2010). This is well known as the TalbotPlateau law (Nelson \& Bartley, 1964; Stockman \& Plummer, 1998). Following this law, and because of the differences in the physical luminances between the flickers, the temporally local average of stimulus luminance should deviate maximally from the global average of luminance around the time of transition between two above-CFF flickers (Fig. 1c).

To avoid confusion, we define several terms regarding stimulus luminance. (1) The flicker amplitude is the difference in luminance between the two components of a flicker stimulus - that is, the peak-to-peak amplitude. Because a flicker stimulus consists of a bright component (we name it "ON frame") and a dark component ("OFF frame"), the amount of flicker amplitude can be described by the difference
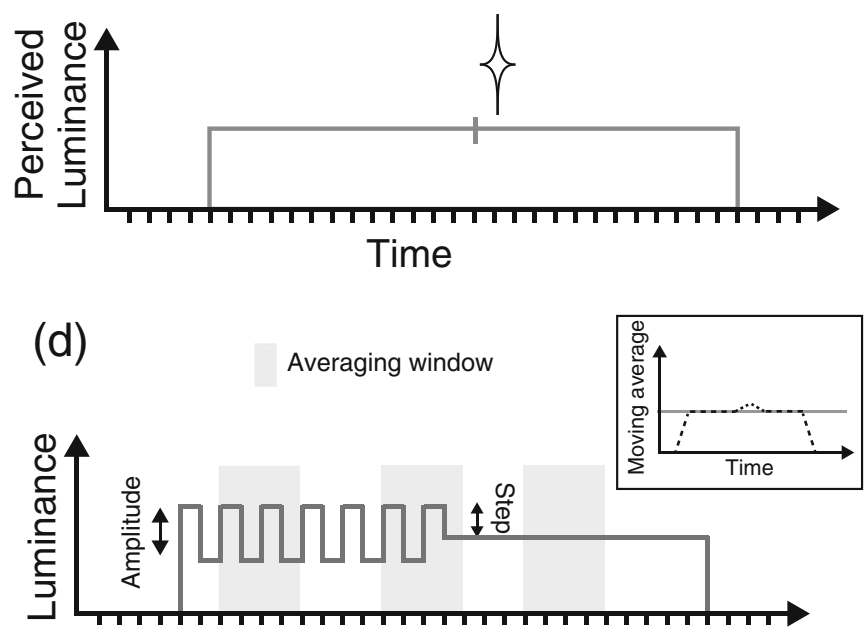

Time

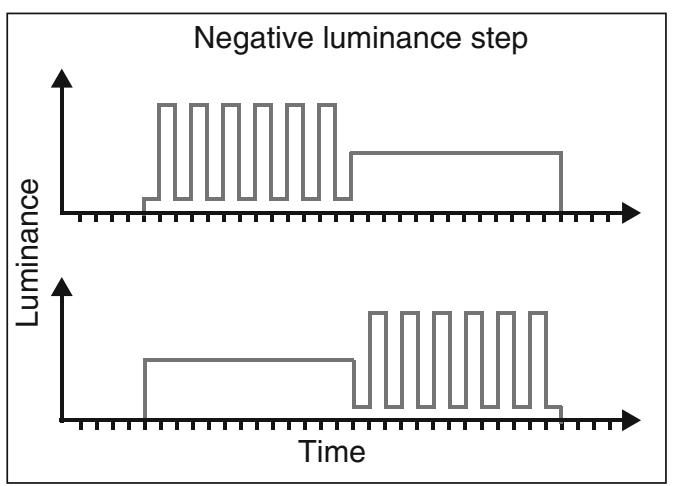

transition should deviate from that either before or after the transition, as is plotted in the side box. (d) A smaller luminance step leads to less temporal change of the moving average around the time of transition. (e) This panel depicts the two types of luminance steps (positive/negative). The polarity of the step was defined by which luminance component (ON/OFF frame) in the flicker stimulus was adjacent to the continuous stimulus 
between these frames. In this study, we set the luminance of the ON and OFF frames independently (described in detail in Exp. 1). (2) The luminance step is the difference between the luminance component in a flicker stimulus and that of a continuous stimulus. The relationship between the flicker amplitude and the luminance step is illustrated in Fig. 1c and d. A flicker stimulus with less flicker amplitude yields a smaller step, and vice versa. As we described above, the components in flicker stimuli can be classified as an ON frame or an OFF frame. Thus, we can also define two luminance steps, one for an ON frame and the other for an OFF frame. We call the former a "positive luminance step" and the latter a "negative luminance step." Note that the concept of luminance step does not simply denote the difference in luminances between the ON/OFF frames of a flicker stimulus and a continuous stimulus, but also is involved with their temporal proximity. That is, the positive (negative) luminance step means that the continuous stimulus precedes or follows the ON (OFF) frame of flicker stimuli (Fig. 1e).

We postulated that perceptual luminance is determined by the temporal moving average of the physical luminances of the stimuli. Under this assumption, the flicker and continuous stimuli would both be perceived as steady-state stimuli with the same perceptual luminance, because the moving averages of their physical luminances are the same, which is consistent with the conventional view- that is, the Talbot-Plateau law (Nelson \& Bartley, 1964; Stockman \& Plummer, 1998). However, if the stimuli are sequentially presented, the moving average should deviate around the time of transition, and we hypothesized that this deviation of the moving average is a plausible cause of TTP (Fig. 1c-d). This hypothesis is consistent with Cheadle et al. (2011), who showed that TTP was attenuated when the flicker amplitude was ramped down, resulting in a smooth transition to the continuous stimuli and a small luminance step.

Several studies have dealt with TPP as an artifact of experimental procedures, because the primary purpose of these studies was to examine whether or not stimuli flickering at the gamma band frequency $(25-70 \mathrm{~Hz})$ could trigger unconscious selective attention (Bauer, Cheadle, Parton, Muller, \& Usher, 2009; Cheadle et al., 2011; van Diepen et al., 2010). Thus, the perceptual mechanism of TTP itself has not yet been directly investigated in these studies. Especially, though Cheadle et al. (2011) suggested that the luminance step would a significant factor of the transient twinkle, they did not actively manipulate its size, but used the same gradual transition between two stimuli. Thus, it is still unclear whether the size of the luminance step affected the occurrence of TTP. When the size of the luminance step is manipulated, the temporal average around the time of transition should be influenced directly (see Fig. 1). This transient deviation can be calculated by using the temporal-averaging model. Comparing the model output and the human perception for various luminance steps would tell us how the simple moving-average model can explain our luminance perception.

The primary objective of this article was to address the effect of fluctuation of the temporal luminance average on TTP. We conducted a simpler version of the experiment to investigate systematically the effect of the luminance step on TTP, and tested whether temporal averaging could consistently explain the TTP. We examined the effects of deviation of the moving average on TTP by manipulating our stimuli in the luminance dimension (Exp. 1) and the temporal dimension (Exp. 2).

\section{Experiment 1}

\section{Materials and method}

Participants Five naïve graduate students (all male) from the University of Electro-Communications and one of the authors (Y.N.) participated in the experiments (age $=24.5 \pm 3.78$ years, range $=22-32$ years). No participants had any neurological or visual disorders, and all had normal or corrected-to-normal vision. Five of the six participants were right-handed. Participants gave their written informed consent, and all experimental protocols were reviewed and approved by the ethical committee of the University of Electro-Communications.

Apparatus Stimuli were generated by the Psychlops software (http://psychlops.sourceforge.jp/; see also Maruya et al., 2010) on a Precision T3500 Workstation (Dell, Inc., Round Rock, TX) with an independent GPU (Quadro FX 1800, NVIDIA Corp., Santa Clara, CA), and displayed on a 21-in. CRT monitor (GDM-F520, SONY Corp., Japan; spatial resolution $=1,024 \times$ 768 pixels; refresh rate $=140,150$, or $170 \mathrm{~Hz}$, depending on the experimental condition). The monitor was gamma-corrected to obtain linear output of luminance. Experiments were conducted in a dark room lit only by the monitor, at a viewing distance of $87 \mathrm{~cm}$ obtained using a chinrest.

Stimuli The stimuli were donut-like rings of different flickering frequencies presented on a gray background $\left(50 \mathrm{~cd} / \mathrm{m}^{2}\right)$. The diameter and width of the ring were 4.0 and $1.0 \mathrm{deg}$, respectively, as is depicted in Fig. 2a. The ring had a sinusoidal luminance profile of 0.5 cycles $/ \mathrm{deg}$, whose minimum luminance was the same at the background (i.e., $50 \mathrm{~cd} / \mathrm{m}^{2}$ ) and the central hole (i.e., the minimum luminance always emerged at the inner/outer edges on the ring and was constant, irrespective of the maximum luminance on the ring). The maximum (peak) luminance of the sinusoidal profile was determined as follows. For the continuous stimuli, rings with identical luminance profiles were repeatedly presented in synchronization with the vertical sync of the CRT monitor. As was described in note 1 , this stimulus was not perceived as flickering, though it in fact did flicker physically. The peak luminance of these 
(a)

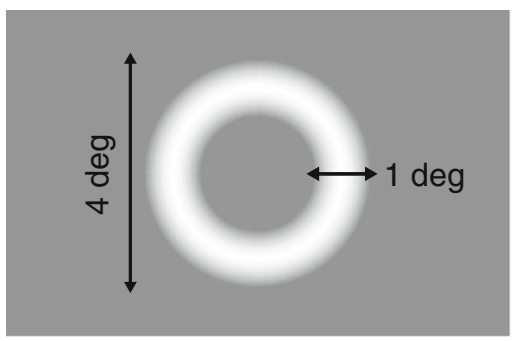

(b)

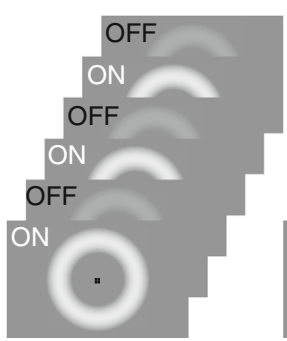

Flicker

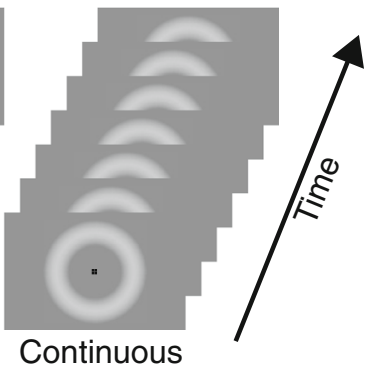

Continuous

(c)

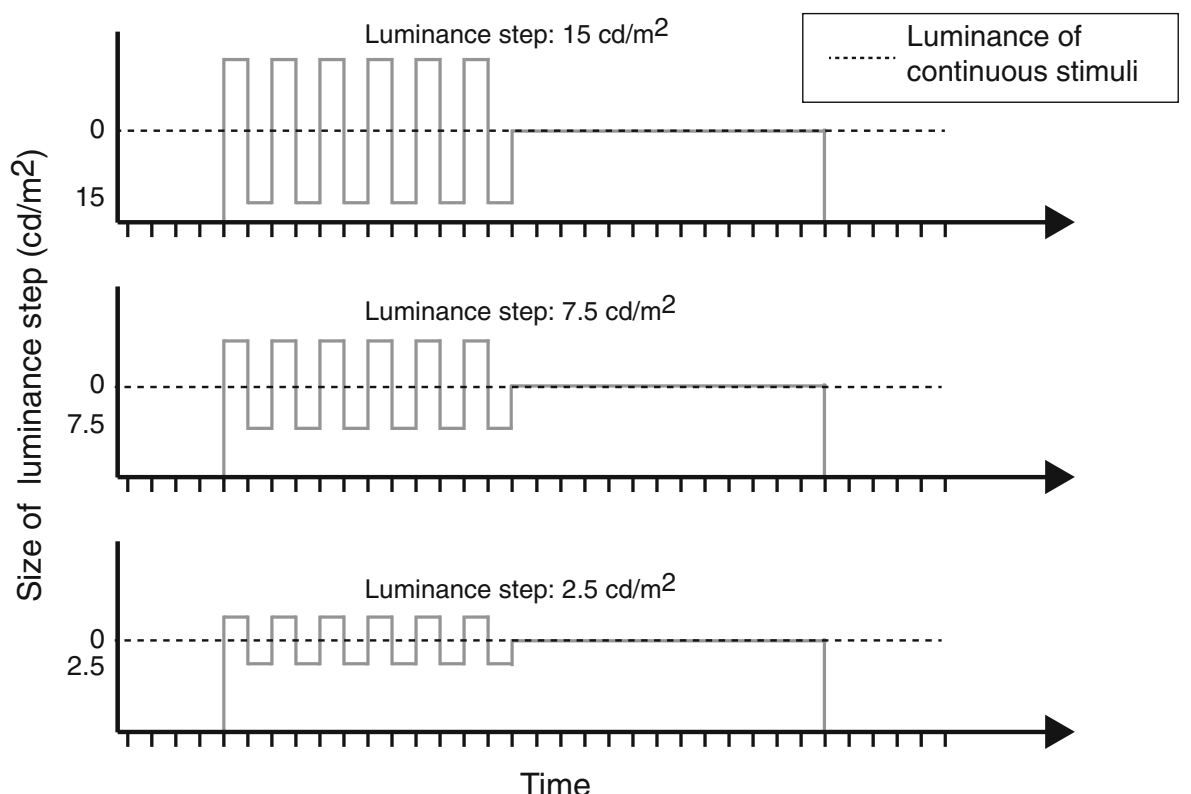

Fig. 2 Schematic diagrams of the stimuli used in Experiment 1. (a) Rings whose luminance had a half-rectified sinusoidal profile were used as stimuli. (b) The flicker stimulus (left) consisted of a bright ring (ON frame) and a dark ring (OFF frame) presented alternately, whereas the continuous stimulus (right) was a stationary illuminant ring. (c) The luminance steps were manipulated by modulating the luminances of the $\mathrm{ON}$ and OFF frames. To equalize the perceptual luminance between flicker and

rings was fixed to $65 \mathrm{~cd} / \mathrm{m}^{2}$ (as a result, the Michelson contrast was 0.13 ). On the other hand, the flicker stimulus was implemented as the alternation of a bright ring ( $\mathrm{ON}$ frame) and a dark ring (OFF frame) (Fig. 2b left). The peak luminances of the $\mathrm{ON}$ and $\mathrm{OFF}$ frames were adjusted during the experimental procedure (described below) so that the perceived luminance (visibility) of the flicker stimulus was equal to that of the continuous stimulus. Note that within a single trial, the flicker amplitude remained constant, unlike in a previous study (Cheadle et al., 2011).

The frequencies of the flicker stimuli were set to 70,75 , or $85 \mathrm{~Hz}$, by means of setting the refresh rate of the CRT monitor to 140,150 , or $170 \mathrm{~Hz}$, respectively. The luminance continuous stimuli, the luminance of the $\mathrm{ON}$ frame was estimated by the PEST method, separately for the individual OFF-frame conditions and participants. The size of the luminance step can be defined by the difference between an OFF (or ON) frame of the flicker stimuli and a luminance component of the continuous stimuli. In panel c, only negative luminance steps of $15,7.5$, and $2.5 \mathrm{~cd} / \mathrm{m}^{2}$ are shown (cf. Fig. 1e)

combinations of the $\mathrm{ON}$ and $\mathrm{OFF}$ frames were determined by the adaptive method described below.

In a trial of Experiment 1, the continuous and flicker stimuli were sequentially presented at identical positions (i.e., the center of the screen). Note that a small fixation dot was presented at the center of the ring to prevent eye movements.

Determining the point of subjective equal luminance Before conducting the main experiment, we confirmed for each participant that the visibilities of the continuous and flicker stimuli were the same. To this end, the point of subjective equal luminance (PSE) between the stimuli was measured individually by the PEST method (Taylor \& Creelman, 1967) separately for each flicker frequency. 
In this measurement, we manipulated the OFF frames of flicker stimuli: Six peak luminances $(50.0,52.5,55.0,57.5$, 60.0 , and $62.5 \mathrm{~cd} / \mathrm{m}^{2}$ ) were used. These conditions created negative luminance steps of $15.0,12.5,10.0,7.5,5.0$, and $2.5 \mathrm{~cd} / \mathrm{m}^{2}$, respectively. In each measurement block, one of the OFF-frame peak luminances was randomly chosen, and it was fixed during the block. On the other hand, the peak luminance of the $\mathrm{ON}$ frame was initially set to $80.0 \mathrm{~cd} / \mathrm{m}^{2}$ (i.e., the positive luminance step was set to $15 \mathrm{~cd} / \mathrm{m}^{2}$ ) and was adaptively adjusted following the PEST procedure, as follows.

In the first interval of a trial, the continuous stimulus was presented for 30 frames as the standard stimulus. After a 200ms blank screen, the flicker stimulus was presented for 30 frames as the comparison stimulus in the second interval. Note that the physical durations for both stimuli varied depending on the flicker frequency (refresh rate). After the presentation of the stimuli, participants indicated which interval included the brighter ring through a two-alternative forced choice (2AFC). When a participant answered that the latter (flicker) stimulus was brighter, the peak luminance of the ON frame in the next trial was lowered, and when the continuous stimulus was brighter, the peak ON-frame luminance of the flicker stimulus was increased. The trials repeated until the adaptive step size was smaller than $1 \mathrm{~cd} / \mathrm{m}^{2}$ (the termination condition). The peak luminance of the $\mathrm{ON}$ frame in the terminated trial was defined as the PSE luminance that induced subjective equal visibility between the continuous and flicker stimuli. A PSE luminance obtained for each OFF-frame peak luminance was also defined as the positive step of the flicker stimulus in the main experiment. As we will show below, the sizes of the positive step were almost identical to those of the negative step.

Procedure of the main experiment In one trial of the main task, two stimulus intervals were sequentially presented, separated by a $400-\mathrm{ms}$ blank frame. One interval (named the "twinkle interval") consisted of a 30-frame continuous stimulus followed by a 30 -frame flicker stimulus. We expected that the twinkle interval could potentially generate TTP due to the switchover of stimuli. The other interval (named the "non-twinkle interval") was a 60-frame continuous stimulus. Two types of twinkle intervals were prepared: One consisted of a positive step to the continuous stimulus, and the other consisted of a negative step (Fig. 1e). The order of the twinkle and non-twinkle intervals was counterbalanced and randomly assigned. In one trial, one of the twinkle stimulus types was also randomly selected (Fig. 2c). Participants were asked to indicate in which interval the twinkle could be perceived (2AFC). The experiment was presented in blocks that differed in flicker frequency $(70,75$, or $85 \mathrm{~Hz})$, and the block order was randomized among participants. In a block, each of the 24 experimental conditions ( 2 orders of twinkle/non-twinkle intervals $\times 2$ types of twinkle stimuli [positive/negative steps] $\times$
6 sizes of luminance step) was randomly presented 20 times, resulting in 480 trials per block.

Procedure of a supplementary experiment A supplementary experiment was also conducted to test whether TTP would be observed if the order of the continuous and flicker stimuli was reversed (the order was fixed in the main experiment). In this experiment, a 30-frame flicker stimulus and a 30-frame continuous stimulus were sequentially presented once, and the order of the flicker and continuous stimuli was randomized trial to trial. The flicker frequency was fixed to $85 \mathrm{~Hz}$, and only a positive-step stimulus was used (Fig. 1e). The size of the luminance step was chosen from among $15.0,7.5$, or $2.5 \mathrm{~cd} / \mathrm{m}^{2}$. Five of the six observers who took part in the main experiment participated, and the task was to answer whether or not TTP was perceived. Each of the six experimental conditions ( 2 presentation orders $\times 3$ sizes of luminance step) was randomly presented 20 times.

\section{Results}

Subjective luminance matching First, we present the PSE luminances of ON-frame luminance obtained by the PEST method. Figure 3 a shows the interparticipant averages of the PSE luminance of the ON frame for different OFF-frame luminances, separated by flicker frequency. The dashed line indicates the predicted PSE luminance satisfying the condition that the ON and OFF frames have equal luminance steps. The PSE luminances of the ON frames are closely aligned to the dashed line, indicating that they increased linearly with the increase in luminance of the OFF frame. This also suggests that the sizes of the positive steps derived from the ON frame should be equal to those of the negative step. Thus, hereafter we refer to each step simply as the "luminance step" (instead of as a "positive" or "negative" luminance step) when we mention the size of a luminance step, except in the discussion of the polarity of the step.

Detection of the transient twinkle We calculated the proportion of trials on which each observer chose the twinkle interval for each condition (TTP ratio), while data were collapsed over the twinkle and non-twinkle intervals. Figure $3 b$ summarizes the interparticipant average TTP ratios for each experimental condition. TTP occurred less often for smaller luminance steps, whereas the flicker frequency and the type of twinkle stimuli apparently had little effect. A three-way analysis of variance (ANOVA: Type of Twinkle Stimuli $\times$ Luminance Step $\times$ Flicker Frequency) revealed a significant main effect of luminance step, $F(5,25)=37.69, p<.0001, \eta^{2}=.50$, and significant interaction between luminance step and flicker frequency, $F(10,50)=3.57, p=.0013, \eta^{2}=.06$. A simple main effect of the flicker frequency was significant for luminance step of $2.5 \mathrm{~cd} / \mathrm{m}^{2}, F(2,10)=7.78, p=.009$; multiple 
(a) Negative luminance step (cd/ $\left.\mathrm{m}^{2}\right)$

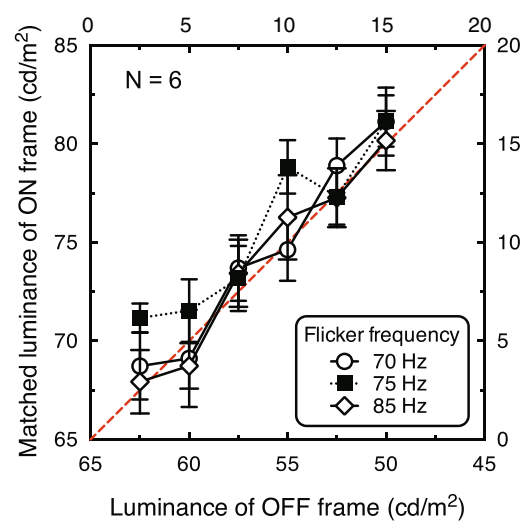

(b)
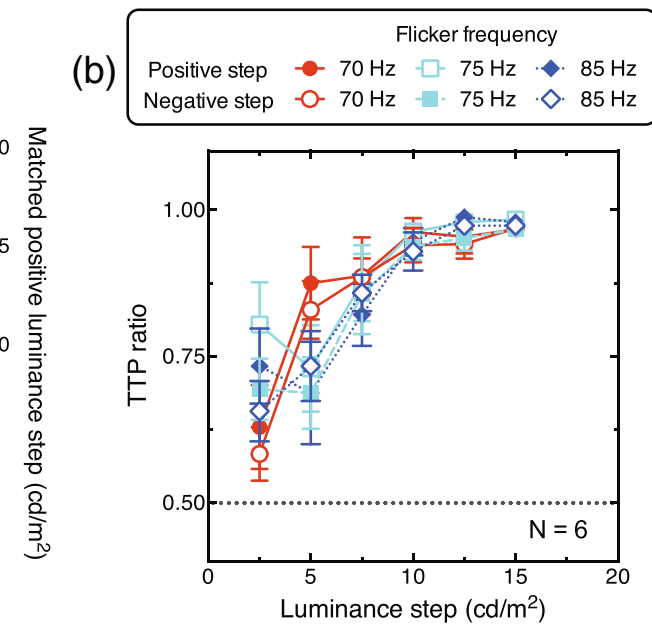

(c)

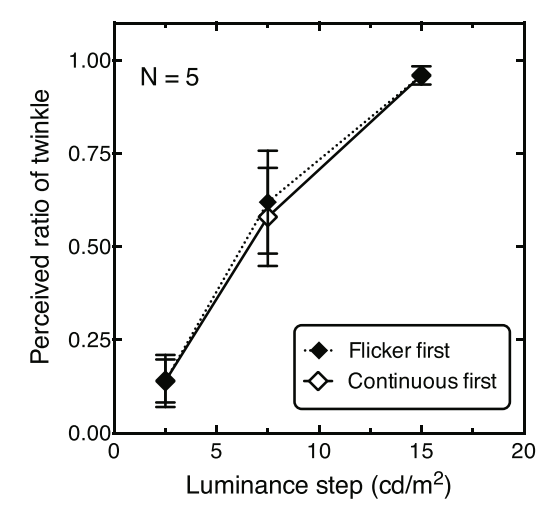

Fig. 3 Results of Experiment 1. (a) Results of subjective luminance matching by the PEST method. The lower horizontal axis indicates the luminance of the OFF frame of the flicker stimulus. The left vertical axis indicates the matched luminance of the ON frame. The dashed line indicates the case in which the luminances between the OFF and ON frames are equal. The corresponding sizes of positive (right vertical axis) and negative (upper horizontal axis) steps are also shown. These sizes are defined by the differences between the luminance of the continuous

comparisons by Bonferroni revealed that the TTP ratio was significantly higher in 75- than $70-\mathrm{Hz}$ conditions $(p<.05$, Bonferroni corrected). A simple main effect of the luminance step was also significant for each frequency: $70 \mathrm{~Hz}, F(5,25)=$ $25.00 ; 75 \mathrm{~Hz}, F(5,25)=14.95 ; 85 \mathrm{~Hz}, F(5,25)=18.06($ all $p \mathrm{~s}$ $<.0001)$. Multiple comparisons with Bonferroni correction revealed that in all frequency conditions, the TTP ratios for 15.0 and $12.5 \mathrm{~cd} / \mathrm{m}^{2}$ were significantly larger than those for $2.5 \mathrm{~cd} / \mathrm{m}^{2}$ (all $p \mathrm{~s}<.05$, Bonferroni corrected).

These results show that little difference was found between the two types of twinkle stimuli. Though the flicker frequencies did affect the TTP ratio for smaller luminance steps, the more decisive factor for TTP was the luminance step; a greater luminance step induced TTP more robustly.

Effect of stimulus order Figure $3 \mathrm{c}$ shows the averaged perceived ratios of TTP, indicating that the presentation order of the flicker and continuous stimuli had no effect on the occurrence of TTP. A two-way ANOVA supported this finding, with no significant main effects for presentation order, $F(1$, $4)=0.08, p=.79, \eta^{2}<.01$, or the interaction, $F(2,8)=0.32, p$ $=.74, \eta^{2}<.01$.

\section{Discussion}

In this experiment, we examined the effect of luminance step on TTP by modulating the flicker amplitude of the flickering stimuli. In contrast to a study in which the flicker amplitude was gradually decreased before the time of transition (Cheadle et al., 2011), here the luminance step was switched instantaneously, which made possible directly investigating the effect

stimulus $\left(=65 \mathrm{~cd} / \mathrm{m}^{2}\right)$ and the luminance of the ON/OFF frame of the flicker stimulus. (b) Results of the main experiment. The averaged transient twinkle perception (TTP) ratios (the ratios at which participants chose the twinkle interval) are plotted as a function of the luminance step. (c) Effect of stimulus (flicker or continuous) presentation order. The horizontal axis indicates the size of the luminance step, and the vertical axis indicates the perceived ratio of the transient twinkle. The error bars in each panel indicate the standard errors of the means

of the luminance step. The results showed that the transient twinkle was more frequently induced by a larger luminance step (Fig. 3b), indicating that the luminance step should be a critical factor for TTP. We also showed that TTP occurs independently from the direction and polarity of the temporal luminance change (Fig. $3 \mathrm{~b}$ and c). These results support our hypothesis that temporal change in the moving average may be a primary cause of TTP. Owing to the luminance step between the continuous and flicker stimuli, the temporal luminance average fluctuates around the time of transition, which could cause TTP.

To examine further the validity of this view, we performed a simple computer simulation with MATLAB (The MathWorks, Inc., Natick, MA) showing how the moving average changes for flicker stimuli over time. In the simulation, the moving average was implemented with a temporally symmetrical Gaussian window. Thus, the moving average can be described by the convolution of the luminance profile of the stimuli and a Gaussian distribution function, as follows:

$m(t)=\sum_{\tau=0}^{T} g(\tau) l(t-\tau)$

where $m(t)$ indicates the moving average at a certain timing $t$, $T$ indicates a window width of the moving average, $g(t)$ indicates the Gaussian distribution function which has a peak at $T /$ 2 and whose sigma is one eighth of $T$, and $l(t)$ indicates the temporal luminance profile of the stimuli. Here, as an example of the simulation, we show the result of a moving average with a 100-ms window width whose $S D$ (of the Gaussian 
distribution) was $12.5 \mathrm{~ms}$. The deviation of the moving average was larger for the larger luminance step (Fig. 4a).

Figure $4 \mathrm{~b}$ summarizes the peak values of the moving average around the time of transition. We applied moving averages with various window widths, ranging from 20 to $180 \mathrm{~ms}$ in 40-ms intervals (Fig. 4b). The relationship between the luminance step and the deviation from the mean luminance ratio (DMR) in the Michelson contrast showed a common tendency, such that the relative amount of deviation increased with larger luminance steps.

To investigate whether the TTP ratio could be explained by the DMR, we transformed the DMR into a probabilistic value by applying the Gamma cumulative distributed function with a shape parameter $k$ and a scale parameter $\theta$. The function gives the relationship between the DMR and the simulated response of TTP. We estimated the window width of the moving average and parameters $k$ and $\theta$ of the function to minimize the error between the averaged TTP ratio and the transformed probability (i.e., the simulated TTP ratio) by the least-squares method. Errors were pooled across experimental conditions. The estimated window width, $k$, and $\theta$ were $120 \mathrm{~ms}, 1.17$, and 0.36 , respectively. We also calculated $R^{2}$, to assess the goodness of fit of the estimated parameters of the function. The resulting

(a)
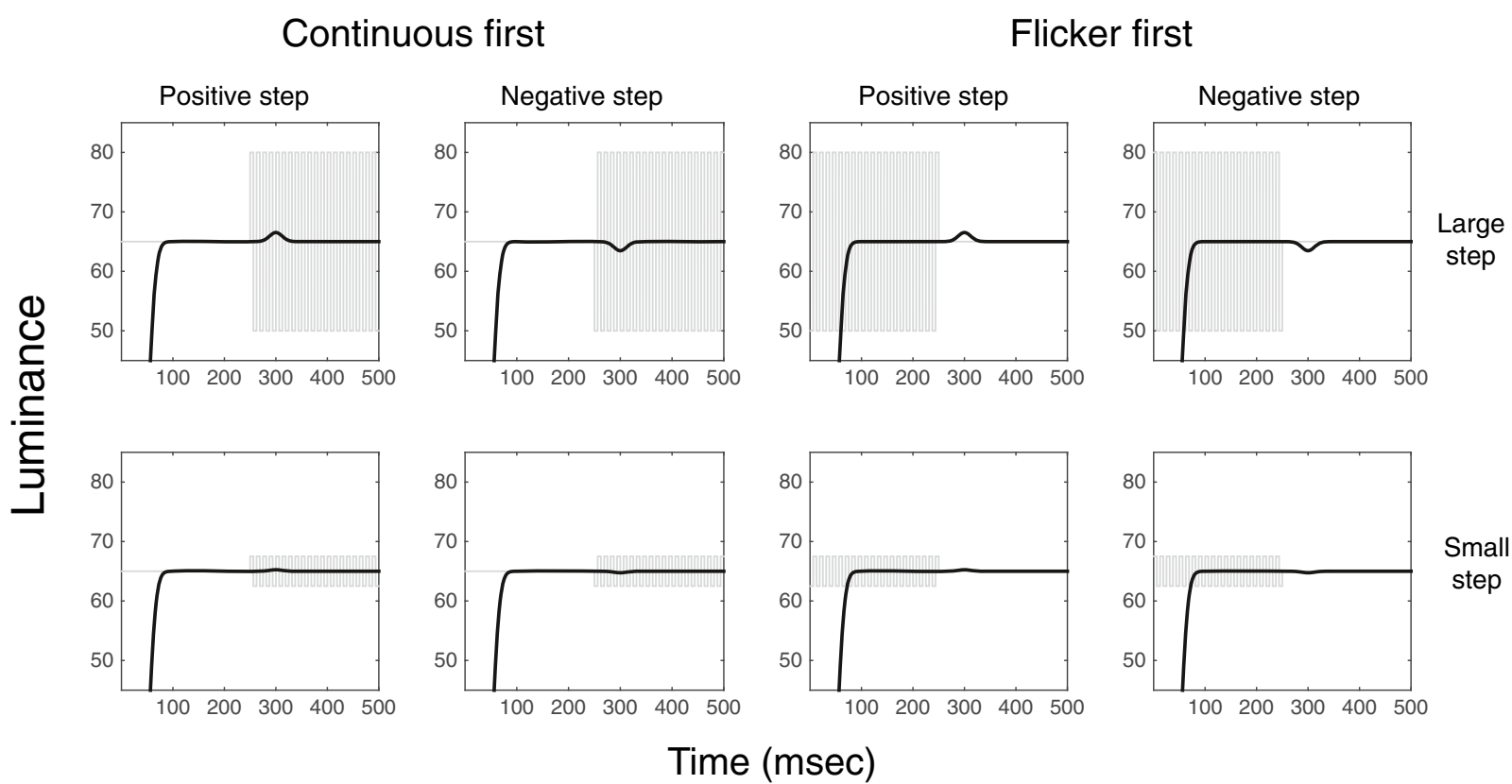

(b)
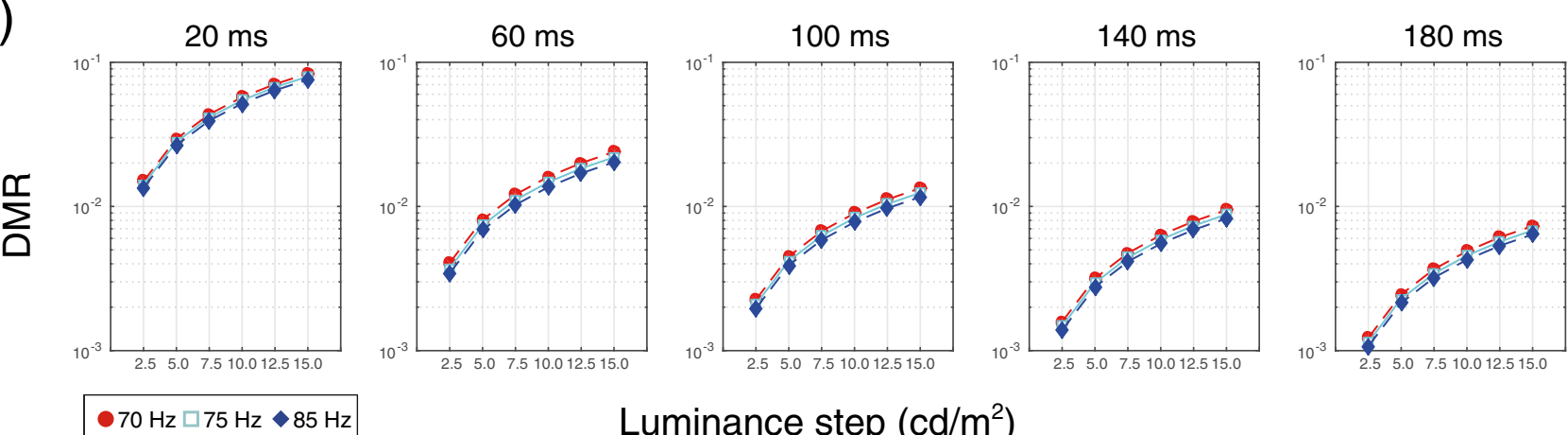

\section{Luminance step $\left(\mathrm{cd} / \mathrm{m}^{2}\right)$}

Fig. 4 Results of a computer simulation of the moving-average model. (a) The thin gray lines in each panel indicate the simulated luminance profile of $85-\mathrm{Hz}$ flicker stimuli. We applied the moving average (details are described in the main text) to the luminance profile, and the output is plotted with a thick curve. Rows indicate different luminance step sizes, and columns indicate the different stimulus transitions (i.e., combinations of different stimulus orders and different step polarities). (b) Contrast between the deviation of the moving average and the mean luminance. The averaging windows have Gaussian profiles with different temporal widths ranging from 20 to $180 \mathrm{~ms}$, in 40-ms intervals. The horizontal axes indicate the sizes of luminance step, and the vertical axes indicate the log contrast between the deviation and the mean luminance (deviations from the mean luminance ratio: DMR). The magnitude of the deviation was estimated by the mean deviation from the mean luminance between 250 and $350 \mathrm{~ms}$ after the transition. Though this figure only shows the results in the condition in which the stimulus order was from a continuous to a flicker stimulus and the luminance step polarity was positive, the same tendency was observed with the reverse stimulus order and with negative luminance steps 
$R^{2}$ s were $.975, .897$, and .947 for 70,75 , and $85 \mathrm{~Hz}$, respectively (Fig. 5).

This similarity between the psychophysical experiment and the computer simulation is consistent with our view that TTP depends on how much the moving luminance average deviates from its long-term mean. In Experiment 2, we manipulated the deviation of the moving average by combining the flicker stimuli with different frequencies and inserting extra OFF frames at the transition to test our moving-average model in the temporal dimension.

\section{Experiment 2}

In Experiment 1, we manipulated the difference in luminance between continuous stimuli and the (components of) flicker stimuli (i.e., luminance step). The result showed that the TTP ratio was higher with a greater luminance step. In a computer simulation, a local moving-average model successfully replicated the occurrence of TTP for different sizes of luminance steps. When the size of the luminance step was sufficiently large, TTP could be induced irrespective of the temporal frequency of the stimuli.

If the moving-average hypothesis is correct, however, the deviation of the luminance average should be affected not only by the luminance step, but also by the width of the averaging window; if a large amount of luminance alternation were included within a wide window, the deviation should not stand out from the long-term mean. Therefore, the window width for temporal averaging is an important parameter for TTP. In other words, the occurrence of TTP would be affected by how frequent a luminance alternation is assigned within the widow.
To investigate the issue, we should manipulate the number of luminance alternations by changing the temporal frequencies between different flicker stimuli having the same flicker amplitude. Deviation of the moving average can be induced by the temporal structure of a visual stimulus. When two flicker stimuli with different flicker frequencies but the same flicker amplitude (i.e., their luminances are perceived as equal) are presented sequentially, the temporal moving average should deviate around the time of their transition, since the numbers of ON and OFF frames change from before to after the transition (cf. Bird \& Mowbray, 1969; Mowbray \& Bird, 1969). It also means that the amount of deviation should vary due to the duration of the ON and OFF frames within the temporal window of the moving average.

The results of Experiment 1 demonstrated the effect of the luminance deviation induced by the luminance step on TTP; however, it remained unclear how the temporal characteristic affects the occurrence of TTP. In Experiment 2, therefore, we investigated TTP from the viewpoint of temporal characteristics, in order to further examine the validity of the moving-average model. We presented two flicker stimuli having different flicker frequencies with the same flicker amplitude, as is described above. In addition, we also inserted additional OFF frames- that is, ISI framesin order to manipulate the temporal property of the stimulus. These manipulations should also induce a large deviation in the moving average around the time of transition as well as our manipulation of flicker amplitude - that is, the luminance step - in the previous experiment. If our hypothesis is correct, TTP should be observed even when successive flicker stimuli with or without an ISI are presented, as long as a certain amount of deviation of luminance average arises around the time of transition. Moreover, we will conclude that TTP should occur due to deviation of the

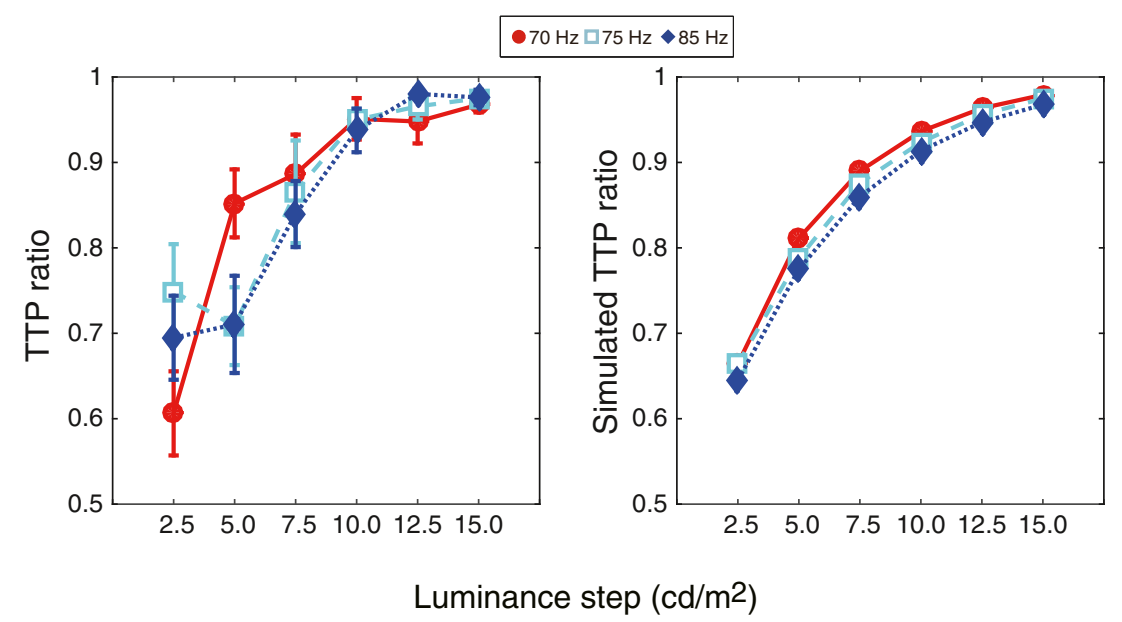

Fig. 5 Transformation of DMR (Exp. 1). The left panel indicates the results of Experiment 1 as a function of luminance step. Error bars indicate the standard errors of the means. The right panel indicates the

transformed DMR (simulated TTP ratio) by applying the Gamma cumulative distribution function with optimized parameters 
luminance average around the time of transition within a temporal averaging window.

For manipulating both the temporal frequency of the flicker stimuli and the ISI, we adopted a high-speed DLP projector that can present binary images with a refresh rate up to $5000 \mathrm{~Hz}$. By making use of this device, we could examine the effect of deviation of the moving average on TTP in hightemporal-frequency conditions that could not be examined with the conventional CRT monitors used in Experiment 1.

\section{Materials and method}

Apparatus Stimuli were projected onto a distal screen (width $=123.5 \mathrm{~cm} \times$ height $93 \mathrm{~cm}$, height from floor to bottom of screen $=113 \mathrm{~cm}$ ) using a high-speed DLP LED projector (DLP Light Commander, Texas Instruments, Inc., Dallas, TX; spatial resolution: $1,024 \times 768$ pixels) in a dark room. Five projector refresh rates were used: 400, 600, 800, 1000, and $1200 \mathrm{~Hz}$. Participants sat in a non-reclining chair about $200 \mathrm{~cm}$ from the screen. Each participant was asked to adjust the chair so that his eye level matched the vertical center of the screen, and also to straighten the spine against the back of the chair to maintain the correct eye position. No chinrest was used. The projector was controlled by Psychlops software (Maruya et al., 2010) and the API for the projector (distributed by Texas Instruments, Inc.) on a MacBook Pro (Apple, Inc., Cupertino, CA). To display the images, we first prepared 1-bit bitmap images and then converted them into the projectorspecific format (.dbi). Next, the converted images were sent to the memory of the projector in advance of starting the experimental session. During the experiment, the order of the preloaded images was controlled by the API.

Participants Four graduate students (all male) in the University of Electro-Communications and author Y.N. participated in the experiment (age $=26.6 \pm 3.61$ years, range $=$ 23-33 years). Three of the participants had also taken part in Experiment 1. None had neurological or visual disorders, and all had normal or corrected-to-normal vision.

Stimuli The stimulus configuration was the same as in Experiment 1; however, because the stimuli were 1-bit bitmap images, each ring had a homogeneous luminance (white or black; Fig. 6a). We modulated the sequence of white ring (W) and black ring (B) frames to display the flicker with different temporal frequencies - that is, flicker stimuli with high temporal frequency (HTF stimuli) and that with low temporal frequency (LTF stimuli). Unlike the transient light emission on a CRT monitor, each ring was presented continuously over a frame. To distinguish the stimulus ring from the background spatially and temporally, 1- by 1-pixel white-andblack checkerboard patterns were presented on the background, so that the perceived background luminance was the mean luminance between the white and black rings.

For the HTF stimuli, one flicker cycle was composed of four frames: WWWB or BWWW. For the LTF stimuli, one flicker cycle consisted of eight frames: WWWWWWBB or BBWWWWWW. Because each frame was displayed synchronously with the refresh rate of the projector, the flickering frequencies of the HTF and LTF stimuli can be regarded as one quarter and one eighth of the refresh frequency, respectively. Thus, the frequency of the HTF stimuli was always twice as high as that of the LTF stimuli. Five HTF stimulus frequencies were used: 100, 150, 200, 250, and $300 \mathrm{~Hz}$, corresponding to the 400-, 600-, 800-, 1000-, and 1200-Hz refresh rates of the projector, respectively. The duty ratios of HTF and LTF stimuli were both $75 \%$ (Fig. 6b), meaning that in a single cycle the temporally averaged luminances of the HTF and LTF stimuli were $75 \%$ of the white-frame luminance. The reason that we selected the $75 \%$ duty ratio was due to only using the 1-bit bitmap images. If the flicker stimuli had been assembled by a number of cycles consisting of the same numbers of $\mathrm{W}$ and $\mathrm{B}$ frames (i.e., $50 \%$ duty ratio), the averaged luminance of the rings would have been the same as the background luminance.

Procedure of the main experiment The general procedure was the same as in Experiment 1. In each trial, two stimulus intervals (twinkle interval and non-twinkle interval, each lasting $320 \mathrm{~ms}$ ) were presented sequentially (separated by $480 \mathrm{~ms}$ ), and participants indicated which interval included the twinkle via a two-alternative forced choice. The twinkle interval was randomly assigned either before or after the blank frame.

In the twinkle interval, the HTF and LTF stimuli were presented sequentially. More specifically, we put a single black ring (B) frame between the two stimuli (Fig. 6c and d, top graphs). We represent the frame sequence of the first stimulus as BWWW (HTF) or BBWWWWWW (LTF), and that of the following stimulus as WWWWWWBB (LTF) or WWWB (HTF). Also, we call the former stimuli $H T F-L T F$ and the latter stimuli $L T F-H T F$. The ISI between the two stimuli was fixed to one frame in which a single B frame was inserted, and this was called the $1 B$-frame condition. We also included $2 B$ - and $3 B$-frame conditions to manipulate the ISI duration (Fig. 6c and d, middle and bottom graphs). The order of the HTF and LTF stimuli in the twinkle interval was counterbalanced. Note that the absolute duration of the ISI for the 1B-frame condition changed depending on the refresh rate. In the non-twinkle interval, only the HTF stimuli (WWWBWWWBWWWB ... .) were sequentially presented for $320 \mathrm{~ms}$. The experiment was presented in blocks that differed in their flicker frequencies, and block order was counterbalanced among the participants. In a block, each of the 12 experimental conditions $(2$ orders of twinkle intervals $\times$ 
(a)
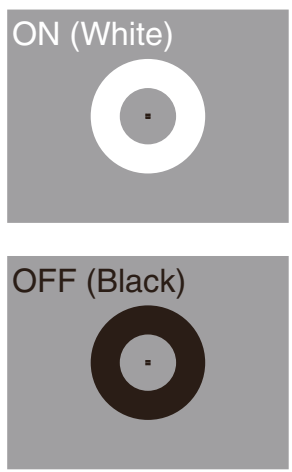

(c) HTF $\rightarrow$ LTF

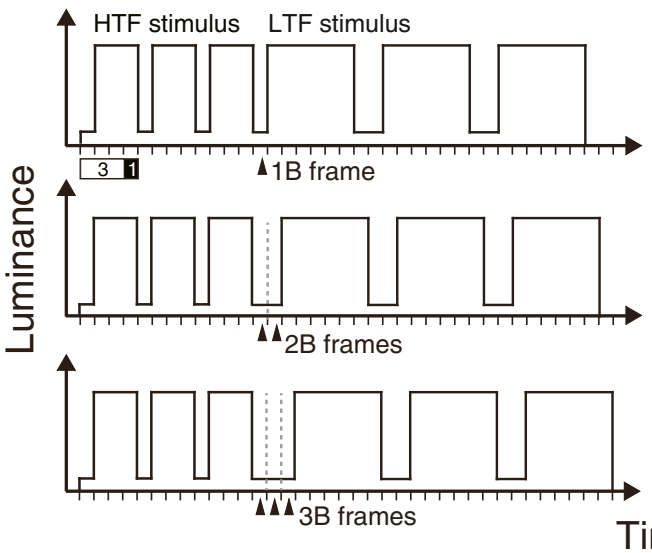

Fig. 6 Stimuli used in Experiment 2. (a) We used homogeneously luminant white and black rings. The background of each stimulus was a checkerboard pattern of $1 \times 1$ pixels. (b) Owing to our high-speed DLP projector, each frame was displayed at a much higher refresh rate than is possible on a CRT monitor, resulting in the temporal frequency of the flicker being one $n$th of the refresh rate. One flicker cycle was composed of four (top, high-temporal-frequency [HTF] stimulus) or eight (bottom,

2 presentation orders for HTF/LTF stimuli $\times 3$ ISIs) was randomly presented ten times, yielding 120 trials per a block.

Procedure to test the perceived luminances To confirm that the perceived luminances of the HTF and LTF stimuli matched, we also conducted a temporal 2AFC experiment examining the difference in subjective luminance between these flickers. The standard stimulus was an HTF stimulus (as in the main experiment: 4 frames/cycle, $75 \%$ duty ratio, $\mathrm{W}: \mathrm{B}=3: 1$ ), and the comparison stimulus was a flickering ring of 8 frames/cycle. The duty ratio of the comparison stimulus was either $25 \%, 62.5 \%, 75 \%, 82.5 \%$, or $100 \%$ (W:B $=2: 6$, $5: 3,6: 2,7: 1$, or $8: 0$, respectively). Here, the stimulus with a $75 \%$ duty ratio was the same as the LTF stimuli, and the other stimuli were fillers to ease the judgment of the task. The standard (and comparison) stimulus was randomly assigned to appear in either the first interval $(320 \mathrm{~ms})$ or the second $(320 \mathrm{~ms})$, and the blank duration between intervals was
$480 \mathrm{~ms}$. The participants were asked to indicate which interval contained the brighter ring. This experiment was also presented in blocks that differed in the flicker frequency of the HTF stimulus. The block order was randomized among participants. In a block, ten trials were assigned for each condition, and thus the number of trials was 100 ( 2 intervals $\times 5$ duty ratios of comparison stimuli $\times 10$ trials).

\section{Results and discussion}

Perceived luminance of the HTF and LTF stimuli We calculated the proportion of trials on which the comparison stimulus was perceived as being brighter than the standard stimulus for each observer. Figure 7a shows the average response ratios among participants in the condition in which the stimuli had the same duty ratio $(75 \%)$. The result revealed that stimuli with identical duty ratios produced the same subjective luminance, even if the temporal frequencies of flicker were 
(a)

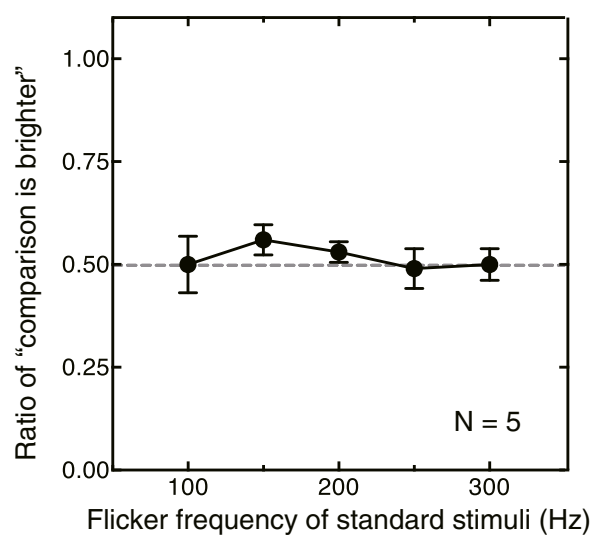

(b)

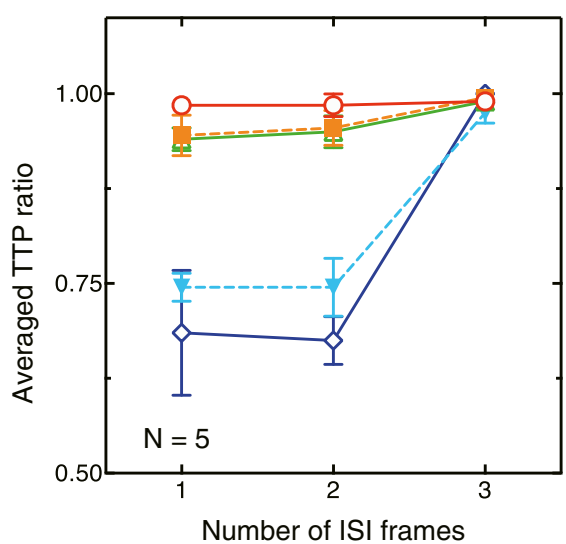

(c)

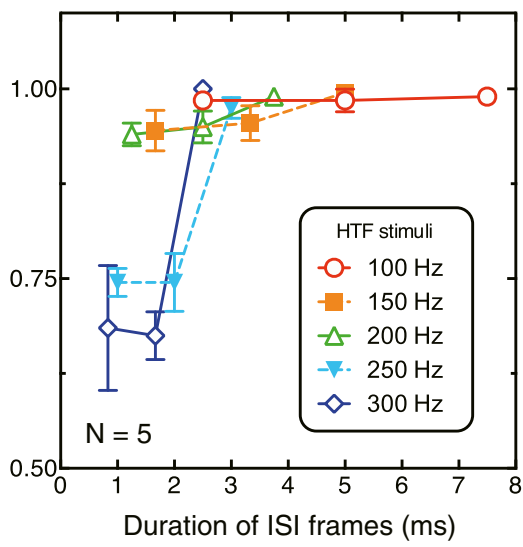

Fig. 7 Results of Experiment 2. (a) Results of the comparison experiment for luminance between the HTF (standard) and LTF (comparison) stimuli. The dashed horizontal line indicates chance level. (b) Averaged
TTP ratios are plotted as a function of the number of ISI frames. (c) They are replotted as a function of the absolute duration of the additional black frames. Error bars in all graphs indicate the standard errors of the means different. This indicates that the perceptual luminances of the HTF and LTF stimuli were successfully matched for each flicker frequency.

TTP ratios for HTF/LTF stimuli We pooled the data from the conditions with counterbalanced orders (twinkle/nontwinkle intervals and presentation orders for the $\mathrm{HTF} /$ LTF stimuli). We plotted the averaged TTP ratios among participants as a function of the number of black frames (Fig. 7b). The panel shows that the TTP ratios were almost maximal in all ISI conditions up to $200 \mathrm{~Hz}$, but were lower in the 1B- and 2B-frame conditions than in the 3B-frame condition when the flicker frequency was at least $250 \mathrm{~Hz}$. Because the duration of a single frame varied with the refresh rate, we assumed that the TTP ratios might be plotted simply as a monotonically increasing function of the physical time, not the frame duration. Thus, we further replotted the results as a function of the ISI in terms of physical time (Fig. 7c). However, that assumption was not true. Specifically, although the TTP ratio was maintained high for ISI durations of $2 \mathrm{~ms}$ or less in the condition of 150 - or $200-\mathrm{Hz}$ HTF, it decreased markedly when the frequency of the HTF stimuli was 250 or $300 \mathrm{~Hz}$. This suggests that the physical time of the ISI frames could not fully describe the occurrence of TTP.

A two-way ANOVA (Flicker Frequency $\times$ ISI) revealed that the interaction between the two conditions was significant, $F(8,32)=11.49, p<.0001, \eta^{2}=.20$. The main effects of flicker frequency, $F(4,16)=23.97, p<.0001, \eta^{2}=.40$, and ISI, $F(2,8)=33.95, p<.0001, \eta^{2}=.22$, were also significant. Analysis of the simple main effects revealed a significant difference of TTP between ISIs for $250 \mathrm{~Hz}, F(2,8)=26.53, p=$ .0003 , and for $300 \mathrm{~Hz}, F(2,8)=18.40, p=.001$. A significant difference of TTP between flicker frequencies also emerged in the 1B-frame ISI, $F(4,16)=13.44, p<.0001$, and the $2 \mathrm{~B}$ frame ISI, $F(4,16)=27.02, p<.0001$. Multiple comparisons with Bonferroni corrections for the difference between the ISIs of 250 and $300 \mathrm{~Hz}$ revealed that the TTP ratios in the $1 \mathrm{~B}$-frame and 2B-frame ISI conditions were significantly lower than those in the 3B-frame conditions (all $p \mathrm{~s}<.05$, Bonferroni corrected). As for the difference between flicker frequencies in the 1B- and 2B-frame ISI conditions, the TTP ratios for 250 and $300 \mathrm{~Hz}$ were significantly lower than those in the other frequency conditions (all $p \mathrm{~s}<.05$, Bonferroni corrected).

Application of the moving-average model To investigate the cause of the difference that occurred for ISI durations (Fig. 7), we ran a computer simulation of the moving average for an HTF stimulus presented with different blank intervals (Fig. 8a). The flicker frequency was chosen from among 100, 120, 150, 200, 240, and $300 \mathrm{~Hz}$. A positive deviation from the mean was observed around the time of transition in the 1B-frame condition, whereas negative deviations were observed in the $2 \mathrm{~B}$ - and $3 \mathrm{~B}-$ frame conditions. This polarity difference of the deviations stemmed from the ratio between the $\mathrm{W}$ and $\mathrm{B}$ frames within the window of the moving average; the computational simulation for the HTF-LTF stimuli suggests that the stimulus around the transition contained more W frames than B frames in the 1-B condition, whereas it contained more $\mathrm{B}$ frames in the 2-B and 3-B conditions. For every HTF stimulus, the deviation size was smaller with higher flicker frequencies and with shorter ISIs.

The magnitudes of the largest deviations in all conditions are summarized in Fig. 8b. The vertical axes (deviations from the mean luminance ratios: DMRs) 
(a)
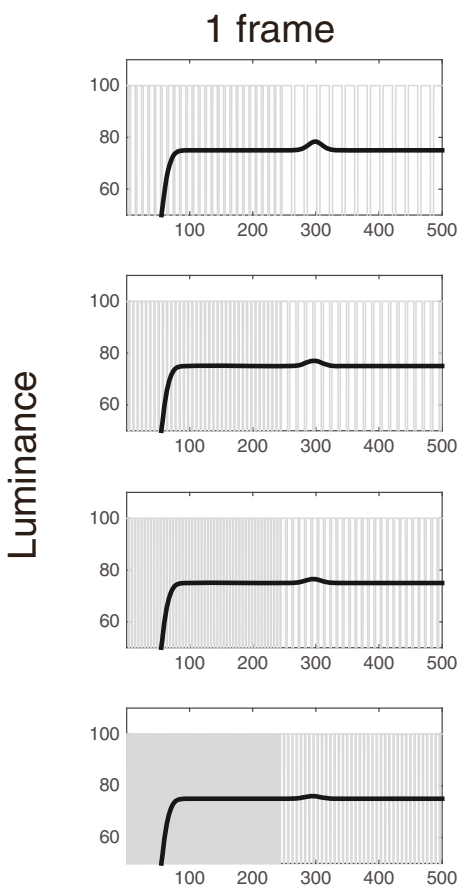

(b)
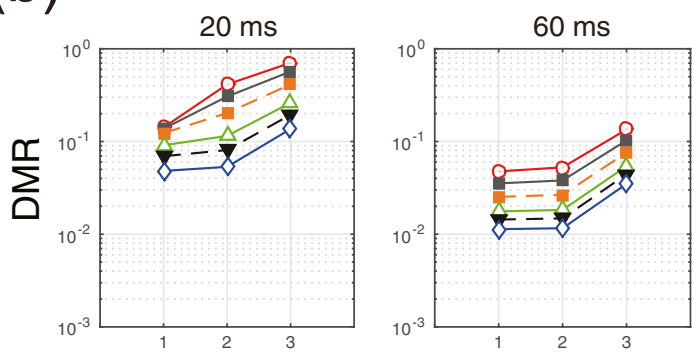

$0100 \mathrm{~Hz}=120 \mathrm{~Hz}-150 \mathrm{~Hz}$ $\triangle 200 \mathrm{~Hz} \nabla 240 \mathrm{~Hz} \diamond 300 \mathrm{~Hz}$

Fig. 8 Results of a computer simulation of the moving averages for HTF and LTF flickers. (a) Thin gray lines in the panels indicate the simulated luminance profiles of 100-, 150-, 200-, and 300-Hz HTF stimuli. We applied a moving average to the profile, and the result is plotted with thick curves. Rows denote the frequencies of the HTF stimuli, and

are plotted on a logarithmic scale. For each ISI, the plotted lines were shifted parallel except for the simulation with a $20-\mathrm{ms}$ window width. This indicates that the differences in the DMRs between frequencies were almost the same on a logarithmic scale. As in Experiment 1, we transformed the DMR into a probabilistic value by applying the Gamma cumulative distributed function. The window width of the moving average and parameters $k$ and $\theta$ were estimated by the least-squares method. Here the estimation was conducted for the 100-, 150-, 200-, and 300-Hz conditions because of the correspondence between the simulation and the psychophysical experiments. After the estimation, we applied the transformation to the remaining frequency
ISI
2 frames
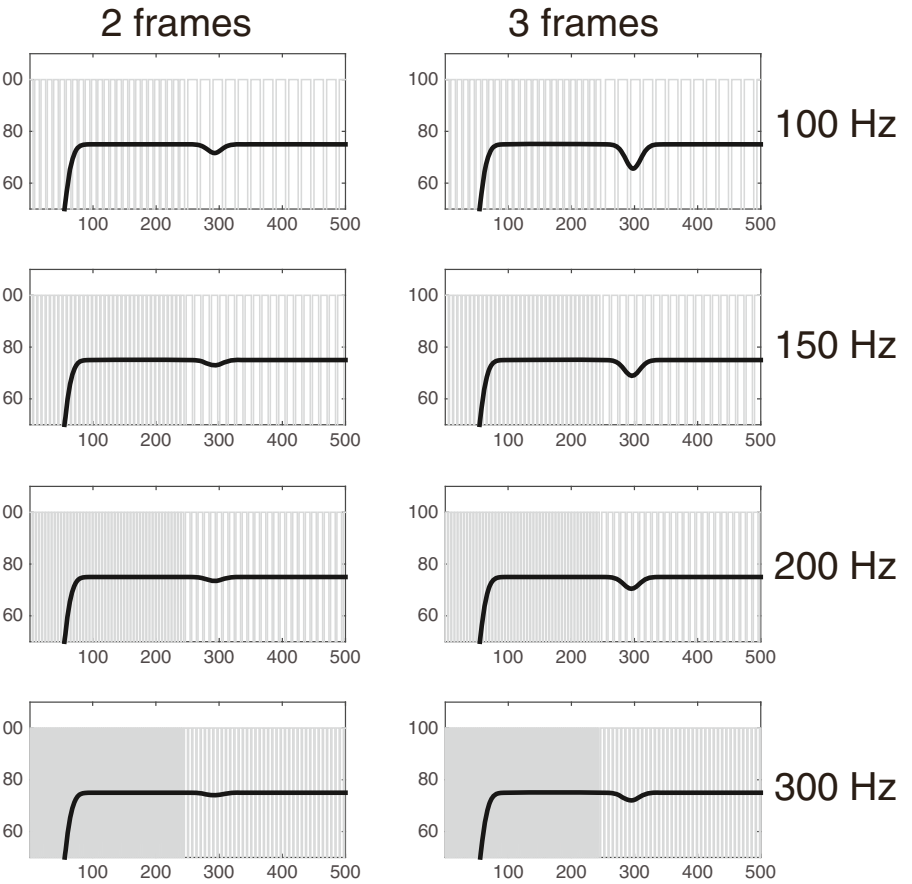

Time (ms)
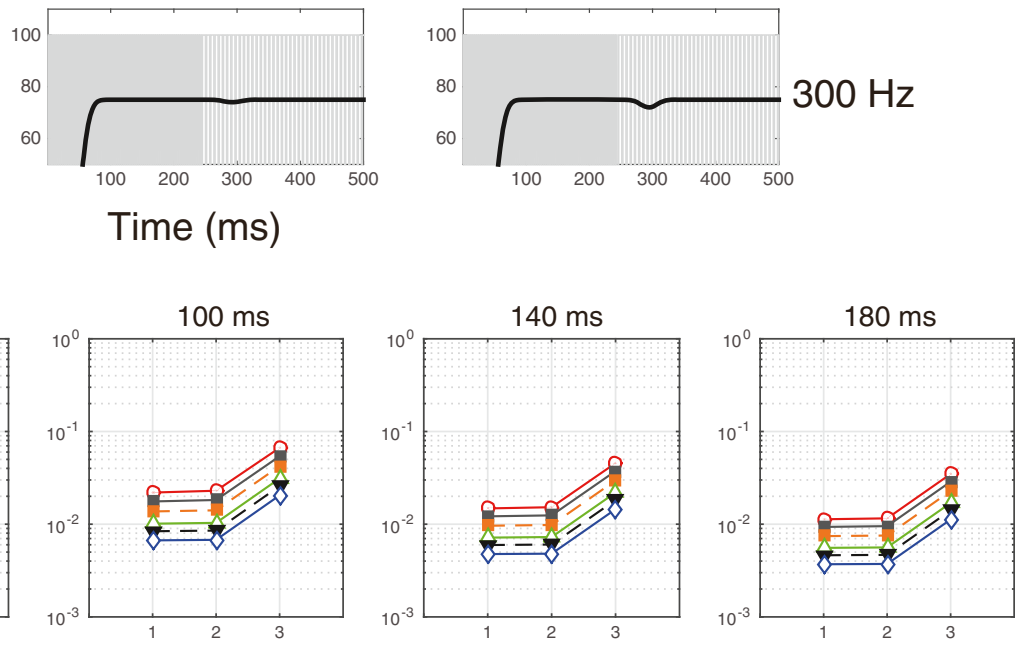

columns indicate the ISI frame number. (b) Deviation of the moving average. To obtain the DMR, we performed the same procedure that was used for producing Fig. 4. The horizontal axes indicate the number of ISI frames, and the vertical axes indicate DMR

conditions with the estimated parameters. The estimated window width, $k$, and $\theta$ were $120 \mathrm{~ms}, 7.45$, and 0.08 , respectively. The $R^{2} \mathrm{~s}$ for each estimated curve were .9166 , $.997, .995$, and .999 for $100,150,200$, and $300 \mathrm{~Hz}$, respectively (Fig. 9).

These results also support our hypothesis that TTP is induced when the moving average of luminance deviates from its long-term mean. The deviation was successfully transformed into DMR, because the DMR was quite similar to the results in both experiments, and the procedures to obtain the DMR were exactly the same between the two experiments. This also implies that the detection of transient changes in the moving average 

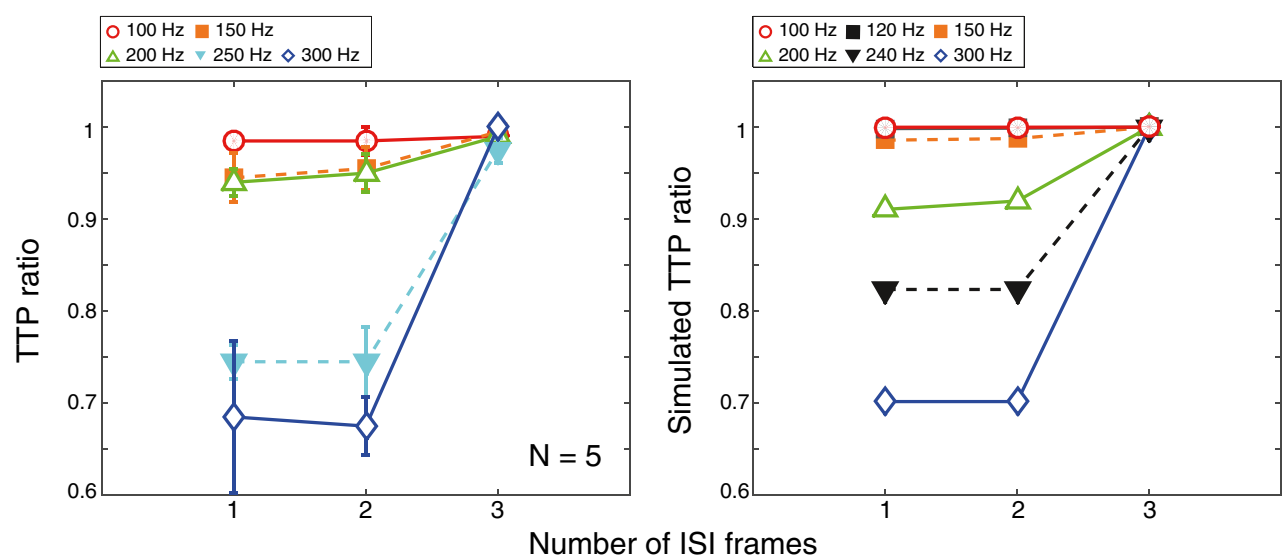

Fig. 9 Transformation of DMR (Exp. 2). The left panel indicates the results of Experiment 2 (and is the same as Fig. 7b), and the right panel indicates the transformed DMR (simulated TTP ratio) by applying the

might be implemented by two processing stages: the linear moving average and nonlinear transformation.

\section{General discussion}

\section{Summary of results}

In this article, we focused on the TTP that occurs around the time of transition between two different stimuli having temporal frequencies higher than the CFF. We showed that a greater luminance step could enhance TTP (Exp. 1), and that flicker up to $200 \mathrm{~Hz}$ (above the CFF) could induce TTP, but that flicker above $200 \mathrm{~Hz}$ could not (Exp. 2). Additionally, for flicker stimuli of 250 and $300 \mathrm{~Hz}$, TTP could be induced by inserting ISI frames between the flicker stimuli (the 3B-frame condition in Exp. 2). These characteristics of TTP were explained with a simple computational model consisting of a temporal-averaging filter and a nonlinear transformation. Both altering the flicker amplitude and inserting ISIs between the stimuli caused the moving average around the time of transition to deviate significantly from the mean luminance, resulting in TTP. Below, we discuss a possible mechanism for TTP from the viewpoint of the moving average in luminance perception.

\section{The explanation of the TTP by the moving-average model}

The experiments demonstrated that TTP was induced by the flicker stimuli at frequencies much higher than the CFF, successfully replicating the results of other studies (Bird \& Mowbray, 1969; Cheadle et al., 2011; Mowbray \& Bird, 1969; van Diepen et al., 2010). Beyond this, our results show that the temporal limit for TTP might be around $200 \mathrm{~Hz}$ (Exp. 2). Our data clearly show that some kinds of transient twinkle percepts could be obtained even for above-CFF stimuli.
Gamma cumulative distribution function with optimized parameters. Note that the conditions of 120 and $240 \mathrm{~Hz}$ were not used in Experiment 2

One might argue that it is contradictory that a transition from a $100-\mathrm{Hz}$ flicker to a $200-\mathrm{Hz}$ flicker can induce robust TTP when we cannot perceive a constant flicker at $100 \mathrm{~Hz}$. This discrepancy can be resolved by the moving-average model, because the moving average depends on whether or not the transition occurs within the temporal range of averaging. For example, if a bright stimulus abruptly appears on a dark background, the moving average (around the stimulus onset) significantly increases, which induces a "bright" perception. If the stimulus disappears, the average should decrease toward the background luminance, which induces a "dark" perception. An intermittent repetition of this stimulus produces repetitive alternation of bright and dark (or background) luminances, leading to the perception of a flicker. With a wider window of temporal averaging, both $\mathrm{ON}$ and OFF frames are included in the averaging window, and the temporal fluctuation becomes smaller. As an extreme, when the fluctuation is almost zero, we can no longer perceive the flicker. However, if the luminances of the ON frames are different between the stimuli before and after the time of transition, then the average should fluctuate around the time of transition, even when the moving averages within the individual flicker stimuli are stationary (and have identical values). Therefore, even if a moving average with a certain window width does not fluctuate for $100-\mathrm{Hz}$ flicker, it could fluctuate at the switch from a 100 - to a $200-\mathrm{Hz}$ flicker.

We can think of several mechanisms for the TTP. Our visual system has not only a low-pass temporal filter (i.e., the moving-average operator), but also a band-pass temporal filter (Kelly, 1961, 1971; Rovamo, Raninen, \& Donner, 1999). One might argue that a better model could be developed by means of combining these filters. Although the processing for TTP might depend on these filters, our simple one-stage averaging model successfully explained our experimental results (Figs. 5 and 9). In our model, a moving average was calculated by averaging the raw (i.e., not filtered) flicker stimuli up to 
$300 \mathrm{~Hz}$. These above-CFF signals might be mediated by the filters in the visual system. As has been shown in previous studies on the steady-state visual evoked potentials for above-CFF flicker (Herrmann, 2001; Lyskov et al., 1998; Ramos-Júnior et al., 2011), our visual system can show neural responses to these temporal signals, irrespective of whether the responses lead to an explicit perception or only to behavior within the neural system. Related to this point, recent studies have pointed out the contribution of above-CFF flicker for conscious perception; the luminance contrast sensitivity function was shifted toward a higher-temporal-frequency region after adaptation to flicker stimuli (Shady, MacLeod, \& Fisher, 2004). Interestingly, the sensitivity was greater than those without adaptation, even when the adaptation flicker was invisible (above CFF) for observers. Johnston et al. (2008) reported a perceptual shrinkage of duration for $10-\mathrm{Hz}$ flicker stimuli after adaptation to above-CFF flicker, suggesting that this effect would be caused by change in the magno cells in the retina and/or in lateral geniculate nucleus. Although we cannot reach a definitive conclusion about the source of TTP, we can at least say that a one- stage moving-average model with a nonlinear transformation can explain TTP.

\section{Temporal characteristics of TTP}

The results of Experiment 2 (Fig. 9 left) suggest the existence of a categorical difference in TTP ratios between $200-\mathrm{Hz}$ and 250-Hz HTF in short-ISI conditions. The simulation by the moving-average model (Fig. 9 right), however, simply showed a gradual decrease of the TTP ratios for these ISIs. This discrepancy might stem from a difference in flicker frequencies between the psychophysical experiments and the computer simulations; we used 100,150,200, 250, and $300 \mathrm{~Hz}$ in the psychophysical experiments, whereas we chose $100,120,150,200,240$, and $300 \mathrm{~Hz}$ for computational convenience. Comparing the $240-\mathrm{Hz}$ condition with the $250-\mathrm{Hz}$ condition, the TTP ratio was slightly lower in the $250-\mathrm{Hz}$ condition, indicating that the TTP ratio at short ISIs gradually decreases with higher flicker frequency.

Related to this issue, the temporal threshold for flicker perception might be much higher than we have known. Recently, by using a similar high-speed device, Davis, Hsieh, and Lee

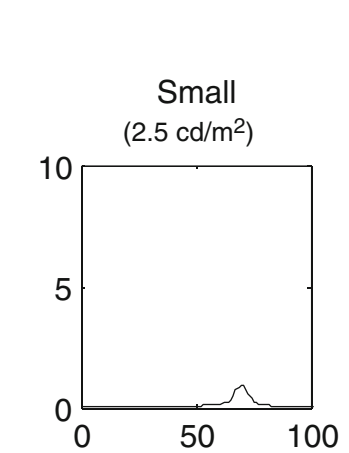

\section{Luminance step}
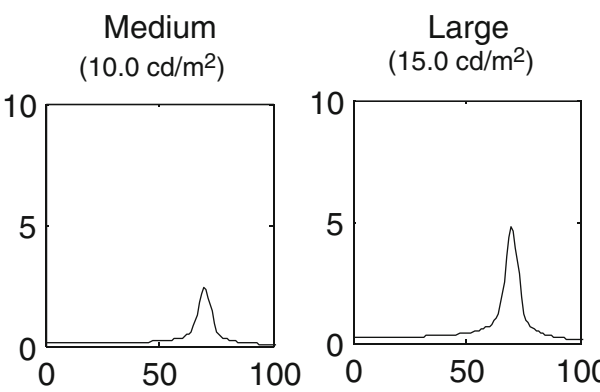

$70 \mathrm{~Hz}$
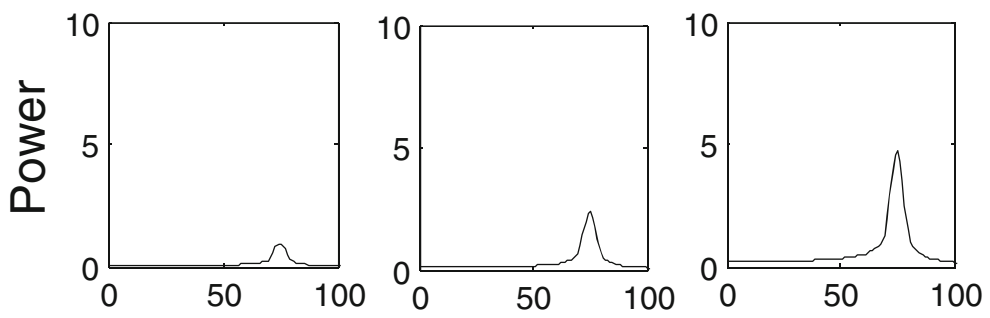

$75 \mathrm{~Hz}$
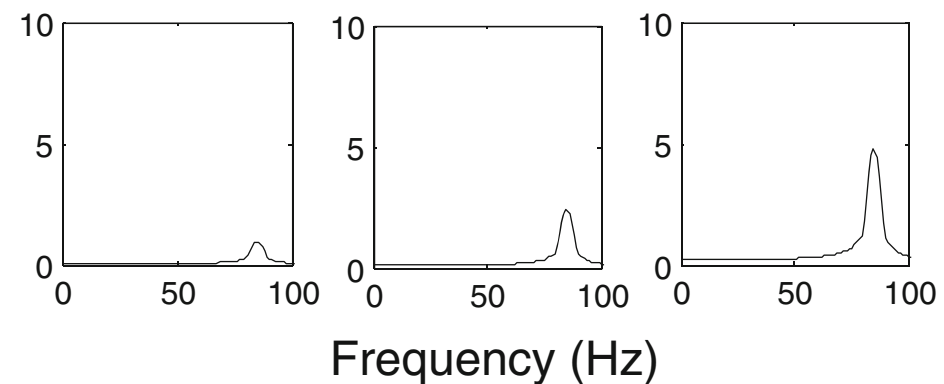

$85 \mathrm{~Hz}$

Fig. 10 Spectrum power of the stimuli (Exp. 1). We applied fast Fourier transforms (FFT, with a Hanning window) to the temporal luminance profiles of the stimuli used in Experiment 1 (cf. the gray lines in
Fig. 4a). Rows indicate different flicker frequencies, and columns indicate different luminance steps. The horizontal and vertical axes of each panel indicate the frequency and the spectrum power, respectively 
(2015) reported that an above-CFF flicker stimulus could be perceived when it had edges with high spatial frequency; they showed that the contrast sensitivity for a flicker stimulus gradually decreased up to around $400 \mathrm{~Hz}$, and drastically dropped off beyond this frequency (see Fig. 3 of Davis et al., 2015). This suggests that our visual system might have another baseline for temporal sensitivity above the CFF. When the flicker frequency of HFT stimuli was $300 \mathrm{~Hz}$, that average TTP ratio was slightly below the differential threshold (.75) for the short ISIs, implying that $300 \mathrm{~Hz}$ might not be the temporal limit for TTP. Therefore, if we examine HTF stimuli with frequencies greater than $300 \mathrm{~Hz}$, we might find the temporal limit of TTP at which the TTP ratio is at chance level (.5). This topic is beyond the present scope, but the high-speed device could possibly reveal high-speed temporal processing that we have not yet found.

\section{Effects of below-CFF frequency components on TTP}

Another possible cause of TTP might be the existence of lowfrequency components in our flicker stimuli. To test this, we applied fast Fourier transforms to the temporal luminance profile of the stimuli, and found that the power of below-CFF frequencies was surely included in the above-CFF flicker stimuli; the power of each frequency component was not zero, but was much lower than the maximum power in the stimuli
(Figs. 10 and 11). Note that below-CFF power exists in both the HTF and LTF stimuli themselves, since our stimuli were square-wave flicker (cf. Fig. 3c of van Diepen et al., 2010).

Although this tendency can also be found in each panel in the third column of Fig. 11, the TTP ratios in the condition of a combination of $150-\mathrm{Hz}$ LTF and $300-\mathrm{Hz}$ HTF were significantly different (Fig. 7c), regardless of the similarity between their spectrum profiles. Moreover, we should note that flickering could not be perceived for either the flicker (Exp. 1) or the HTF or LTF (Exp. 2) stimulus alone, although these stimuli contained considerable power in the below-CFF frequency region. This indicates that the existence of below-CFF frequency components could not directly be linked to stimulus perception, and thus, the power of below-CFF frequencies must not be the direct cause of TTP.

\section{Perceived brightness of TTP}

If a moving average directly denoted the perceptual luminance, the nature of TTP might depend on the direction (or polarity) of deviation from the mean luminance. Our results, however, did not support this idea. The results of Experiment 1 (Fig. 3b and c) showed that TTP was consistently observed irrespective of the stimulus order (continuous $\rightarrow$ flicker or flicker $\rightarrow$ continuous). Additionally, in Experiment 2, TTP occurred almost equally for positive and negative deviations (Fig. 7). Computer simulations

$|S|$
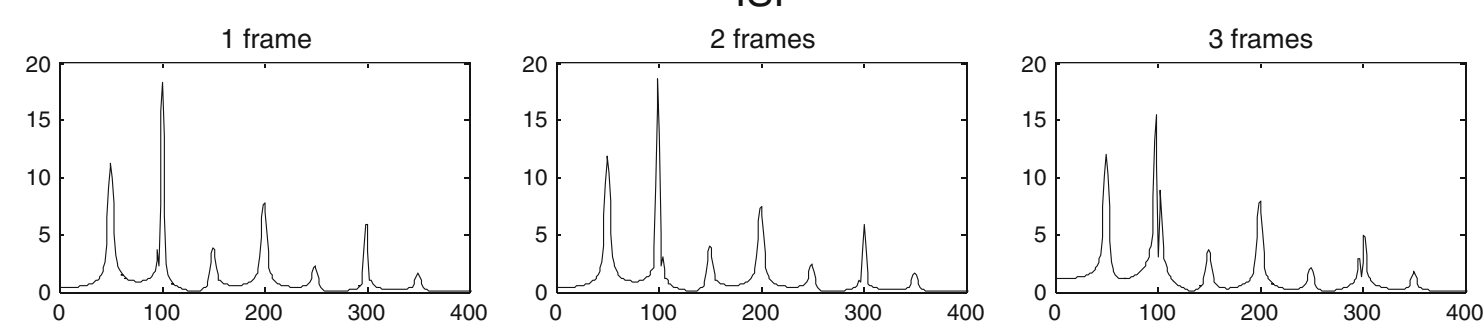

$50 \mathrm{~Hz} \rightarrow>100 \mathrm{~Hz}$
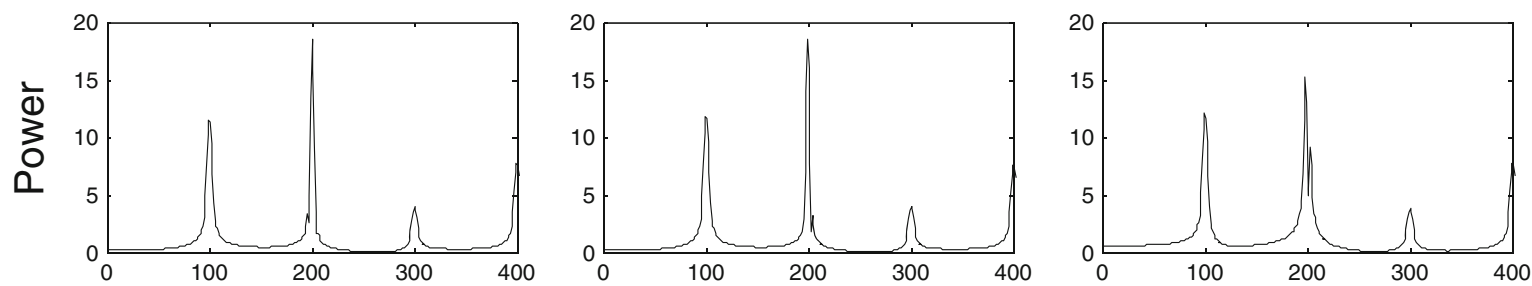

$100 \mathrm{~Hz} \rightarrow 200 \mathrm{~Hz}$
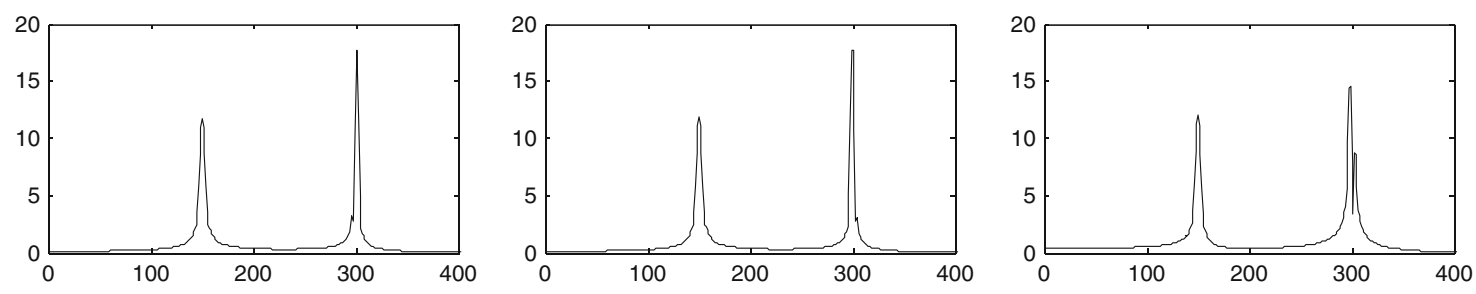

Frequency $(\mathrm{Hz})$

Fig. 11 Spectrum power of the stimuli (Exp. 2). We applied FFT to the temporal luminance profile of the stimuli used in Experiment 2 (cf. the gray lines in Fig. 8a). Rows indicate different LTF-HTF combinations, and columns indicate different numbers of ISI frames. The horizontal and vertical axes of each panel indicate the frequency and the spectrum power, respectively 
of the moving-average model showed good correspondence to these results when we calculated the absolute values of deviation (Figs. 5 and 9). A previous study had reported that the perceived luminance of a transient flash varied among individuals; one participant could discriminate the brightness of a flash, and some could not (Mowbray, Flower, \& Bird, 1975). We also confirmed that it was very hard even for an experienced observer to differentiate the TTP luminance induced by negative or positive luminance steps.

One possible explanation for this is that the important factor for TTP might be independent of the polarities of luminance changes. This view seems consistent with findings regarding the perception of luminance change. First, Roufs (1974) indicated that incremental and decremental brief luminance flashes cannot be distinguished. Second, a decremental probe presented on a flickering background is perceived as being the same as an incremental probe (Wolfson \& Graham, 2001). These findings support the idea that the polarity of deviation does not greatly affect the perceived luminance of TTP. This characteristic would be an advantage to detect the transient change in the environment as quickly as possible, without a careful examination of the contents in the change. If, for TTP, our visual system might not be sensitive to the luminance polarity of deviation around the transition, the occurrence of TTP would be based on absolute variation from the luminance average. The neural responses for the transient and flicker signals should be shown in the magnocellular pathway (for a review, see Livingstone \& Hubel, 1988). Thus, TTP, especially the detection of the deviated luminance average, might be processed in this pathway. The magno cells of macaques show maximal sensitivity to $10-\mathrm{Hz}$ flickering stimuli (Lee, Martin, \& Valberg, 1989), whose frequency almost corresponds to the estimated window width of the moving average $(120 \mathrm{~ms}, 8.3 \mathrm{~Hz})$. Further research will be needed to test whether or not magnocelluar pathway processing is related to TTP. For example, this issue could be resolved by applying adaptation to flickering stimuli at various temporal frequencies before the observation of TTP stimuli (cf. Chapman, Hoag, \& Giaschi, 2004; Nieuwenhuis, Jepma, La Fors, \& Olivers, 2008). If our estimated window width of moving average reflects the temporal sensitivity of the magnocelluar pathway, the adaptation to flickering stimuli at an optimal temporal frequency would impair the occurrence of TTP.

Another possible explanation of insensitivity to luminance polarity might be the low contrast (DMR) between the longterm average and the deviation of this average. Computational simulations of DMR (Figs. $4 \mathrm{~b}$ and $8 \mathrm{~b}$ ) and simulated TTP ratios (Figs. 5 and 9) suggest that a higher TTP ratio could be shown even for approximately 0.01 of DMR (Figs. 4b and $8 \mathrm{~b})$. The amount of DMR almost corresponds to the highest temporal contrast sensitivity (cf. Kelly, 1961), indicating that the DMR would be near the detection threshold level. If the luminance deviation around the stimulus transition is quite different from the long-term averaged luminance, observers might distinguish the polarity of TTP. For example, we could modify the flicker stimuli used in Experiment 1: If the luminance of the OFF frame were lower than the background luminance, we could display flicker stimuli with a greater amplitude than in Experiment 1-that is, a large luminance step could be presented. In this sense, sensitivity to the polarity of deviation might depend on the polarity of the component luminances of flicker stimuli relative to the background: Whether the component luminance crosses the luminance of background might be critical. In any case, in our experiments the TTP ratios for positive and negative deviations did not show any differences. This suggests that the polarity of the deviation does not affect the occurrence of TTP. If anything, it would not contribute to the luminance perception for TTP, but to perceiving the emergence of deviation itself.

In summary, although we cannot conclude whether TTP may or may not be accompanied by brightness perception, the important factor for TTP is whether a sufficient amount of temporal change in the moving average emerges around the time of transition.

\section{Conclusions}

The present study has shown that TTP is dependent on the size of the luminance step at the transition between stimuli having different luminance components. A larger luminance step results in a larger temporal deviation from the long-term luminance mean. The degree of temporal deviation could explain this phenomenon. The temporal deviation should also be brought about by successive flicker stimuli with different temporal frequencies. The perception of transient twinkles is determined by whether or not the amount of the moving luminance average around the transition exceeds a certain threshold.

Author note This research was supported by the Core Research for Evolutional Science and Technology (CREST) Program "Creation of Human-Harmonized Information Technology for Convivial Society" at the Japan Science and Technology Agency, and by the Japan Society for the Promotion of Science, KAKENHI Grant Number 24830030.

\section{References}

Bauer, F., Cheadle, S. W., Parton, A., Muller, H. J., \& Usher, M. (2009). Gamma flicker triggers attentional selection without awareness. Proceedings of the National Academy of Sciences, 106, 16661671. doi:10.1073/pnas.0810496106

Bird, J. F., \& Mowbray, G. H. (1969). Visual transient phenomenon: Its polarity and a paradox. Science, $165,588-589$.

Chapman, C., Hoag, R., \& Giaschi, D. (2004). The effect of disrupting the human magnocellular pathway on global motion perception. Vision Research, 44, 2551-2557. doi:10.1016/j.visres.2004.06.003 
Cheadle, S. W., Parton, A., Muller, H. J., \& Usher, M. (2011). Subliminal gamma flicker draws attention even in the absence of transition-flash cues. Journal of Neurophysiology, 105, 827-833. doi:10.1152/jn. 00357.2010

Davis, J., Hsieh, Y. H., \& Lee, H. C. (2015). Humans perceive flicker artifacts at $500 \mathrm{~Hz}$. Science Reports, 5, 7861. doi:10.1038/ srep07861

Emoto, M., \& Sugawara, M. (2012). Critical fusion frequency for bright and wide field-of-view image display. Journal of Display Technology, 8, 424-429. doi:10.1109/Jdt.2012.2191390

Forsyth, D. M., \& Brown, C. R. (1961). Nonlinear property of the visual system at fusion. Science, 134, 612-614. doi:10.1126/science.134. 3479.612

Herrmann, C. S. (2001). Human EEG responses to 1-100 Hz flicker: resonance phenomena in visual cortex and their potential correlation to cognitive phenomena. Experimental Brain Research, 137, 346353. doi:10.1007/s002210100682

Johnston, A., Bruno, A., Watanabe, J., Quansah, B., Patel, N., Dakin, S., \& Nishida, S. (2008). Visually-based temporal distortion in dyslexia. Vision Research, 48, 1852-1858. doi:10.1016/j.visres.2008.04. 029

Kelly, D. H. (1961). Visual responses to time-dependent stimuli: I. Amplitude sensitivity measurements. Journal of the Optical Society of America, 51, 422. doi:10.1364/josa.51.000422

Kelly, D. H. (1971). Theory of flicker and transient responses: I. Uniform fields. Journal of the Optical Society of America, 61, 537-546. doi: 10.1364/josa.61.000537

Lee, B. B., Martin, P. R., \& Valberg, A. (1989). Sensitivity of macaque retinal ganglion cells to chromatic and luminance flicker. Journal of Physiology, 414, 223-243.

Levinson, J. Z. (1968). Flicker fusion phenomena. Science, 160, 21-28. doi:10.1126/science.160.3823.21

Livingstone, M., \& Hubel, D. (1988). Segregation of form, color, movement, and depth: Anatomy, physiology, and perception. Science, 240, 740-749. doi:10.1126/science.3283936

Lyskov, E., Ponomarev, V., Sandstrom, M., Mild, K. H., \& Medvedev, S. (1998). Steady-state visual evoked potentials to computer monitor flicker. International Journal of Psychophysiology, 28, 285-290. doi:10.1016/S0167-8760(97)00074-3

Maruya, K., Hosokawa, K., Kusachi, E., Nishida, S., Tachibana, M., \& Sato, T. (2010). A system for rapid development and easy sharing of accurate demonstrations for vision science. Frontiers in Neuroscience Conference Abstract: Neuroinformatics, 2010, 4. doi:10.3389/conf.fnins.2010.13.00093

Mowbray, G. H., \& Bird, J. F. (1969). The simple reaction time as an aid in determining the sign of a visual transient response. Acta Psychologica, 30, 84-95. doi:10.1016/0001-6918(69)90042-0
Mowbray, G. H., Flower, R. W., \& Bird, J. F. (1975). Visual cortex responses to abrupt changes in the periodicity of rapidly intermittent light. Electroencephalography and Clinical Neurophysiology, 39, 305-312.

Nelson, T. M., \& Bartley, S. H. (1964). The Talbot-plateau law and the brightness of restricted numbers of photic repetitions at CFF. Vision Research, 4, 403-411. doi:10.1016/0042-6989(64)90012-4

Nieuwenhuis, S., Jepma, M., La Fors, S., \& Olivers, C. N. (2008). The role of the magnocellular and parvocellular pathways in the attentional blink. Brain and Cognition, 68, 42-48. doi:10.1016/j.bandc. 2008.02.119

Ramos-Júnior, S. G., Celino, D. R., Rodor, F. F., Ribeiro, M. R., \& Muller, S. M. (2011). Experimental evidences for visual evoked potentials with stimuli beyond the conscious perception threshold. In Proceedings of the 2011 Biosignals and Biorobotics Conference (BRC) (pp. 1-5). Piscataway: IEEE Press. doi:10.1109/brc.2011. 5740685

Roufs, J. A. (1974). Dynamic properties of vision: IV. Thresholds of decremental flashes, incremental flashes and doublets in relation to flicker fusion. Vision Research, 14, 831-851. doi:10.1016/00426989(74)90148-5

Rovamo, J., \& Raninen, A. (1984). Critical flicker frequency and $M$ scaling of stimulus size and retinal illuminance. Vision Research, 24, 1127-1131. doi:10.1016/0042-6989(84)90166-4

Rovamo, J., Raninen, A., \& Donner, K. (1999). The effects of temporal noise and retinal illuminance on foveal flicker sensitivity. Vision Research, 39, 533-550. doi:10.1016/S0042-6989(98)00120-5

Sen, T. K. (1964). Visual responses to two alternating trains of highfrequency intermittent stimuli. Journal of the Optical Society of America, 54, 386-393.

Shady, S., MacLeod, D. I., \& Fisher, H. S. (2004). Adaptation from invisible flicker. Proceedings of the National Academy of Sciences, 101, 5170-5173.

Stockman, A., \& Plummer, D. J. (1998). Color from invisible flicker: A failure of the Talbot-Plateau law caused by an early "hard" saturating nonlinearity used to partition the human short-wave cone pathway. Vision Research, 38, 3703-3728. doi:10.1016/S0042-6989(98) 00049-2

Taylor, M. M., \& Creelman, C. D. (1967). PEST: Efficient estimates on probability functions. Journal of the Acoustical Society of America, 41, 782-787. doi:10.1121/1.1910407

van Diepen, R. M., Born, S., Souto, D., Gauch, A., \& Kerzel, D. (2010). Visual flicker in the gamma-band range does not draw attention. Journal of Neurophysiology, 103, 1606-1613. doi:10.1152/jn. 00629.2009

Wolfson, S. S., \& Graham, N. (2001). Comparing increment and decrement probes in the probed-sinewave paradigm. Vision Research, 41, 1119-1131. doi:10.1016/S0042-6989(01)00009-8 